\title{
OS DESASTRES NATURAIS NO BRASIL E SEUS AGRAVOS À POPULAÇÃO: REVISÃO INTEGRATIVA
}

\author{
Sara Larissa de Melo Araújo ${ }^{1}$ \\ Ana Catarina de Melo Araújo ${ }^{2}$ \\ Rayssa Santos Botelho ${ }^{3}$ \\ Rennata Cristina Mariz Pereira4 \\ Maria Tervânia Soares Araújo5 \\ Roberta Barbosa de Lima6 \\ Adriana de Melo Araújo7 \\ Wagner Pereira da Silva8
}

\section{RESUMO}

Desastres naturais consistem em eventos capazes de causar sofrimento e prejuízos a uma população. Tem-se, no Brasil, as inundações, enchentes e deslizamentos que afetam municípios e indivíduos com maior vulnerabilidade social. Objetivou-se identificar os principais agravos à saúde decorrentes dos desastres naturais ocorridos no Brasil. Trata-se de uma revisão integrativa, utilizando o método de Prática Baseada em Evidências (PBE), realizada de janeiro/2013 a dezembro/2018. Como resultados encontrou-se que as áreas mais afetadas ficam na região sul com 1247 municípios. Já referente aos desastres que mais ocorreram deduziu-se que incêndios florestais, secas, inundações, deslizamentos, vendavais e granizo foram os mais prevalentes. os principais fatores que contribuem para os agravos à saúde identificados foram áreas inabitáveis por períodos significativos, comprometimento dos serviços de saneamento ambiental, da quantidade e da qualidade da água, do solo e da oferta de alimentos afetados como também alterações nos ciclos de vetores hospedeiros e reservatórios de doenças e maior incidência de doenças e óbitos. Assim, a identificação dos riscos a desastres naturais deve incentivar ações estruturais, preparação técnica de profissionais de saúde e conscientização da população. Portanto, os resultados obtidos podem ser relevantes para sociedade e profissionais de saúde, para elaboração de estratégias, ações de vigilância, prevenção e mitigação dos riscos e agravos ficam como contribuição.

Palavras-chave: Desastres; Saúde Pública; Vulnerabilidade.

\footnotetext{
${ }^{1}$ Enfermeira Residente em Urgência e Emergência pelo Programa de Residência Multiprofissional em Saúde da Universidade Federal do Vale do São Francisco, Petrolina, Pernambuco.

${ }^{2}$ Enfermeira, Doutorado pela Universidade de Pernambuco. Recife, Pernambuco, Brasil.

${ }^{3}$ Enfermeira Residente em Hematologia pelo Programa de Residência Uniprofissional da Secretaria de

Saúde de Pernambuco, Recife, Pernambuco.

${ }^{4}$ Enfermeira Residente em Nefrologia pelo Programa de Residência Uniprofissional da Secretaria de

Saúde de Pernambuco, Recife, Pernambuco.

${ }^{5}$ Enfermeira pela Universidade Maurício de Nassau, Recife, Pernambuco, Brasil.

${ }^{6}$ Enfermeira pela Universidade Maurício de Nassau, Recife, Pernambuco, Brasil.

${ }^{7}$ Enfermeira, Hospital Esperança e Hospital da Unimed, Recife, Pernambuco.

${ }^{8}$ Enfermeiro mestre em Ciências da Saúde pela Universidade de Pernambuco, Recife Pernambuco, Brasil.
} 


\title{
NATURAL DISASTERS IN BRAZIL AND THEIR HEALTH PROBLEMS: AN INTEGRATIVE REVIEW
}

\begin{abstract}
Natural disasters consist of events capable of causing suffering and harm to a population. In Brazil, there are floods, floods and landslides that affect municipalities and individuals with greater social vulnerability. The objective was to identify the main health problems resulting from natural disasters in Brazil. This is an integrative review, using the Evidence Based Practice (EBP) method, performed from January 2013 to December 2018. As results it was found that the most affected areas are in the southern region with 1247 municipalities. Regarding the most common disasters, it was deduced that forest fires, droughts, floods, landslides, windstorms and hail were the most prevalent. The main factors contributing to the identified health problems were areas uninhabitable for significant periods, impairment of environmental sanitation services, the quantity and quality of water, soil and food supply affected, as well as changes in host vector cycles. reservoirs of disease and higher incidence of disease and death. Thus, the identification of risks to natural disasters should encourage structural actions, technical preparation of health professionals and public awareness. Therefore, the results obtained may be relevant to society and health professionals, for the elaboration of strategies, surveillance actions, prevention and mitigation of risks and injuries are a contribution.
\end{abstract}

Keywords:Disasters; Public health; Vulnerability.

Artigo Recebido em 25/09/2019 e Aceito em 19/12/2019 


\section{IINTRODUÇÃO}

Desastres naturais são acontecimentos capazes de causar sofrimento e significativos prejuízos físicos, moral, material e emocional. Estes, agem como importante fator de disrupção do espaço privado familiar e identidade social o que leva ao limite a experiência e os valores do ser humano e sua vida em sociedade(VALÊNCIO, 2015). Desastres naturais, tais como inundações, enchentes, deslizamentos, erosão, terremotos, tornados, furacões, tempestades, estiagem, entre outros, podem ocorrer em qualquer parte do mundo, embora normalmente as regiões mais afetadas são aquelas com maior vulnerabilidade do sistema social(BODSTEIN, 2014; GUIMARÃES, 2014; PARIZZI, 2014; CONFAONIERI, 2015).

Esses eventos resultam da combinação de quatro fatores a citar: a ocorrência de uma ameaça natural; capacidades ou medidas insuficientes para reduzir os potenciais riscos; os danos à saúde da população, e uma população exposta às condições de vulnerabilidade social e ambiental(FREITAS, 2014).

Referente aos fatores mencionados anteriormente, a vulnerabilidade socioambiental é considerada o fator de maior impacto por ser decorrente de uma combinação de processos sociais relacionados à precariedade de trabalho, saúde, renda, educação, saneamento, entre outros, associados aos desastres naturais provenientes da degradação ambiental tais como desmatamento de encostas e leitos de rios, por exemplo(GUIMARÃES, 2014).

No Brasil, o processo acelerado de urbanização verificado nas últimas décadas, levou ao crescimento das cidades, muitas vezes em áreas inapropriadas à ocupação, aumentando as situações de perigo e de risco a desastres naturais(BRASIL, 2012; SARDINHA, 2016). Inundações severas acompanhadas por deslizamentos de terra, quase sempre resultantes da ausência de políticas públicas de saneamento básico, são os principais desastres registrados no país e precisam ser melhor avaliados e suas causas determinadas a fim de que sejam elaboradas, para cada uma de suas fases, 
Revista Científica do Corpo de Bombeiros Militar de Pernambuco

Artigo Publicado no Vol.06 N.15 - Edição Jan a Jun2020 - ISSN 2359-4829

Versão on-line disponível em: http://www.revistaflammae.com

estratégias eficazes que possam promover maior grau de resiliência(ASSUMPÇÃO, 2017).

Os ciclosdos desastres naturais se dividem em cinco fases, que consistem em: prevenção, mitigação, alerta, resposta e reabilitação/reconstrução. As três primeiras ocorrem antes do desastre e têm como finalidade prevenir que ocorram danos maiores, pois, algumas vezes não é possível evitar sua ocorrência. Nas fases de resposta e reabilitação são realizadas ações para diminuir o sofrimento da população atingida. Nessas fases ocorrem os fatores que contribuem para os agravos em saúde pública(PARIZZI, 2014; MINERVINO, 2016).

Em curto prazo - fase de resposta - há a necessidade de tratar indivíduos com ferimentos leves, moderados e graves, por meio de ações de atendimento pré-hospitalar e resgate. Prazos mais longos - fase de reconstrução - estão relacionados à incidência de doenças transmissíveis como leptospirose e doenças diarreicas, capazes de agravar o quadro clínico de pacientes crônicos, em geral, portadores de hipertensão arterial, doenças cardiovasculares, diabetes, entre outros. Nestes casos, deve-se propor e executar estratégias de vigilância, controle e prevenção de enfermidades, com programas de assistência à saúde, logo após o evento danoso(FREITAS, 2014; COSTA, 2017; CARVALHO, 2016).

Os fatores que contribuem para os agravos à saúde em prazos mais longos ocorrem pelo fato de a ocorrência dos desastres tornar inabitáveis por períodos significativos, áreas que, até então, eram locais de moradia. Este fato requer do estado à tomada de providências de reabilitação em abrigos temporários, e a reestruturação do ambiente físico. As consequências de um desastre comprometem ainda os serviços de saneamento ambiental, a quantidade e a qualidade da água, o solo e a oferta de alimentos, bem como alterações nos ciclos de vetores, hospedeiros e reservatórios de doenças(FREITAS, 2014; CARMO, 2014). 
Ao que se refere as consequências para a saúde, essas são inevitáveis nesses casos, uma vez que as pessoas afetadas, precisam conviver com consequências sobre a infraestrutura, serviços e economia local afetados, tais como a interrupção do atendimento de saúde, efeitos sobre a agricultura e pecuária, o que atinge ainda, a produção e qualidade dos alimentos(COSTA, 2017).

Dessa forma, o conhecimento acerca dos impactos causados pelos desastres naturais é fundamental, para que se possam entender os riscos associados, sobretudo, pelo surgimento de patógenos e enfermidades com significativas consequências para a saúde pública no local de incidência. Assim, o presente estudo, teve como objetivos, compreender quais as regiões do Brasil mais afetadas pelos desastres naturais?, quais os tipos de desastres naturais que ocorrem com maior frequência no Brasil? e quais os principais fatores que contribuem para agravos à saúde decorrentes pelos desastres naturais?

\section{MATERIAIS E MÉTODOS}

Tratou-se de uma revisão integrativa, um dos métodos de pesquisa mais utilizados na Prática Baseada em Evidências (PBE), comum em pesquisas de enfermagem. Este tipo de estudo permite a incorporação das evidências na prática clínica e tem como finalidade reunir e sintetizar resultados de pesquisas sobre um delimitado tema ou questão, de maneira sistemática e ordenada, contribuindo para o aprofundamento do conhecimento do tema investigado(CROSSETTI, 2012).

Procurou-se identificar artigos científicos que respondessem as seguintes questões norteadoras: Quais as regiões do Brasil mais afetadas pelos desastres naturais? Quais os tipos de desastres naturais que ocorrem com maior frequência no Brasil? Quais os principais fatores que contribuem para agravos à saúde decorrentes pelos desastres naturais? 
$\mathrm{Na}$ coleta de dados, foi realizada a busca de artigos científicos na base de dados LILACS (Literatura Latino-Americana e do Caribe em Ciências da Saúde) e na biblioteca virtual SCIELO (Scientific Electronic Library Online). Essa busca utilizou as terminologias cadastradas nos Descritores em Ciências da Saúde - DeCS, criados pela Biblioteca Virtual em Saúde - BVS que permite o uso da terminologia comum em Português, Inglês e Espanhol. Os descritores utilizados na busca foram: desastres, saúde pública e vulnerabilidade. Foi utilizado como ferramenta de auxílio, o operador Booleano "AND", para o cruzamento dos descritores.

Como critério de inclusão foram selecionados artigos disponíveis na íntegra, com textos completos e originais, que continham especificidade com o tema e a problemática do estudo, publicados em português, espanhol e inglês, nos últimos cinco anos de janeiro/2013 a dezembro/2018 por trazerem informações mais atualizadas.

Além disso, foi utilizado o manual do Ministério da Integração Nacional, considerando a importância que ele teve para fundamentação do trabalho. Foram excluídos artigos que não abordaram o objetivo da pesquisa bem como monografias, dissertações, teses, livros e publicações fora do período supracitado, incompletos ou produzidos com dados de outros países.

Foi realizada a leitura na íntegra dos artigos selecionados e retirada as seguintes informações: nome do periódico, título do artigo, autores, cenários, método, objetivo, ano de publicação dos artigos e resultados). Na etapa seguinteas informações foram dispostas em quadros contendo esses tópicos para ajudar na interpretação dos dados, após essa construção foram discutidos os resultados encontrados, os quais foram identificados a partir da leitura dos artigos estudados na íntegra e expostos em forma de tabelas. 
Revista Científica do Corpo de Bombeiros Militar de Pernambuco

Artigo Publicado no Vol.06 N.15 - Edição Jan a Jun2020 - ISSN 2359-4829

Versão on-line disponível em: $\underline{\text { http://www.revistaflammae.com }}$

\section{RESULTADOS E DISCUSSÃO}

Após relacionar os descritores com as características propostas na metodologia do estudo e refiná-los com o operador booleano AND, os cruzamentos dos descritores e os critérios de inclusão e exclusão, foram encontrados 67 (sessenta e sete) artigos relacionados ao tema e préselecionados 12 (doze), como mostra o Quadro 2.

Quadro 1 - Distribuição quantitativa dos artigos encontrados nas bases de dados e de acordo com os descritores utilizados.

\section{Principais fontes utilizadas para pesquisa}

\begin{tabular}{lll} 
Descritores & Scielo & Lilacs \\
Desastres & 1.610 & 547 \\
Saúde Pública & 1.470 & 1913 \\
Vulnerabilidade & 65 & 600 \\
Desastre AND Saúde Pública AND Vulnerabilidade & 67 & 12 \\
\hline Total de artigos referentes a temática: & 67 & \\
Pré selecionados & 12 &
\end{tabular}

Fonte: Dados do estudo.

Os artigos incluídos, nesta revisão, estão descritos conforme nos Quadros 2 e 3: o nome do periódico, título do artigo, autor(es), ano de publicação, objetivos, metodologia aplicada e resultados.

Quadro 2 - Resumo dos estudos pesquisados envolvendo periódico, título e autor.

\begin{tabular}{|c|c|c|}
\hline PERIÓDICO & TíTULO & AUTOR \\
\hline Saúdeem Debate & $\begin{array}{l}\text { Possíveis contribuições da integração das } \\
\text { políticas públicas brasileiras à redução de } \\
\text { desastres }\end{array}$ & Assumpção et al \\
\hline Ambiente\&Sociedade & $\begin{array}{l}\text { A vulnerabilidade do idoso em situações de } \\
\text { desastres: necessidade de uma política de } \\
\text { resiliência eficaz }\end{array}$ & Bodstein et al \\
\hline $\begin{array}{l}\text { Physis-Revista de } \\
\text { Coletiva }\end{array}$ & $\begin{array}{l}\text { Biossegurança e desastres: conceitos, } \\
\text { prevenção, saúde pública e manejo de } \\
\text { cadáveres }\end{array}$ & Cardoso et al \\
\hline $\begin{array}{l}\text { Revista Rede de Cuidados em } \\
\text { Saúde }\end{array}$ & $\begin{array}{l}\text { Desenvolvimento de instrumento para } \\
\text { avaliação dos planos municipais de } \\
\text { contingência de desastres naturais no Estado } \\
\text { do Rio de Janeiro }\end{array}$ & Carvalho et al \\
\hline Terra livre & $\begin{array}{l}\text { Variabilidade climática, vulnerabilidade social e } \\
\text { saúde no Brasil }\end{array}$ & Confalonieri \\
\hline Saúdeem Debate & $\begin{array}{l}\text { Abrigos temporários em desastres: a } \\
\text { experiência de São José do Rio Preto, sil }\end{array}$ & Costa et al \\
\hline Ciência\&SaúdeColetiva & Mortalidade por desastres no Brasil: o que & Carmo e Anazawa \\
\hline
\end{tabular}


Revista Científica do Corpo de Bombeiros Militar de Pernambuco

Artigo Publicado no Vol.06 N.15 - Edição Jan a Jun2020 - ISSN 2359-4829

Versão on-line disponível em: $\underline{\text { http://www.revistaflammae.com }}$

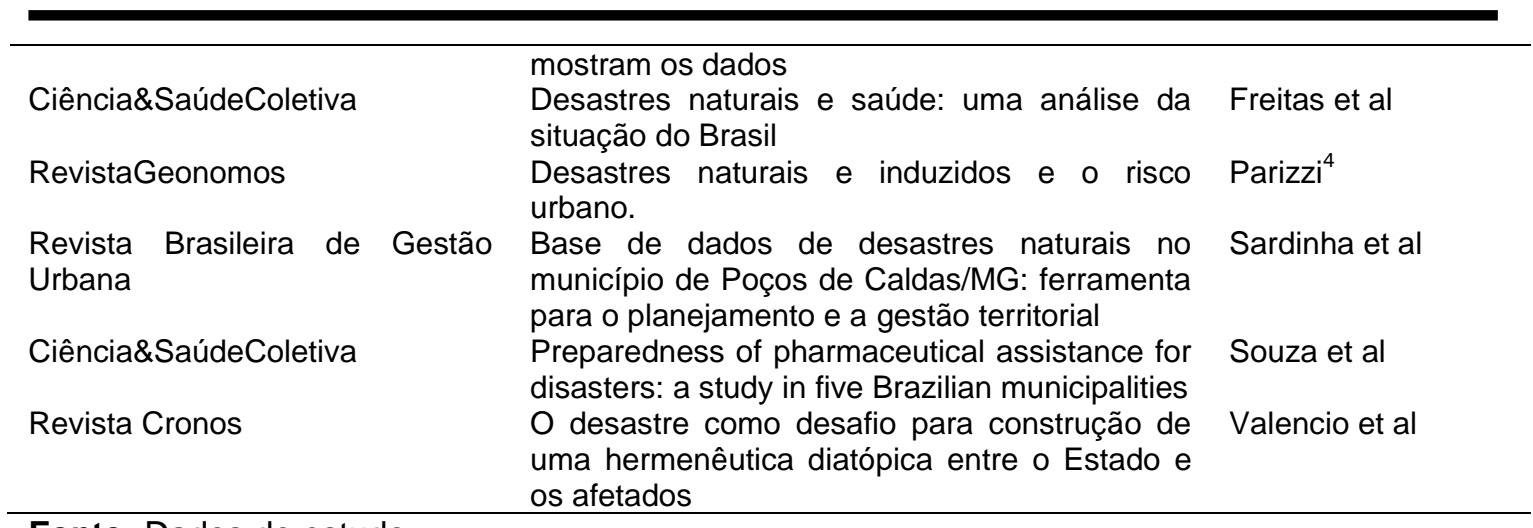

Fonte: Dados do estudo

Os periódicos apresentados no Quadro 2, apontaram para áreas diversas de interesse ao tema abordado por este estudo. Destacaram-se entre as áreas de pesquisa, saúde coletiva, cuidados e debates em Saúde, totalizando $58,3 \%$ das publicações. Outras revistas incluíram questões do ambiente e ciências sociais (Ambiente \& Sociedade) 8,33\%; gestão urbana (Revista Brasileira de Gestão Urbana e Revista Cronos) 16,66\% e produção do conhecimento geográfico (Terra livre e Revista Geonomos) 16,66\%.

Apesar de haver uma área de predomínio do resultado, eram muito importantes trabalhos com visões diversificadas, sobretudo pelo fato das soluções eram obras estruturadoras e sustentabilidade. Os impactos na saúde pública eram as consequências de um planejamento inadequado. Isso ressalta as soluções interdisciplinares.

Quadro 3 - Resumo dos estudos pesquisados envolvendo cenário, método e ano.

\begin{tabular}{|c|c|}
\hline $\begin{array}{l}\text { CENÁRIO } \\
\text { Política Nacional de Recursos Hídricos (Lei } \\
\text { no 9.433/1997), da Política Federal de } \\
\text { Saneamento (Lei no 11.445/2007), da } \\
\text { Política Nacional de Defesa Civil (Lei no } \\
\text { 12.608/2012) }\end{array}$ & $\begin{array}{l}\text { MÉTODO } \\
\text { A metodologia adotada neste artigo tem como } \\
\text { base a pesquisa exploratória, sendo utilizado } \\
\text { como procedimento técnico a pesquisa } \\
\text { documental }\end{array}$ \\
\hline $\begin{array}{l}\text { Lei } 12.608 \text {, de abril de 2012, e no Glossário } \\
\text { de Defesa Civil (1998) }\end{array}$ & $\begin{array}{l}\text { Os procedimentos metodológicos da pesquisa } \\
\text { incluem a coleta de dados na legislação } \\
\text { referente aos direitos do idoso, em relatórios } \\
\text { produzidos por órgãos oficiais, em documentos } \\
\text { internacionais e na literatura que trata do tema } \\
\text { do idoso e questões afins, de modo geral }\end{array}$ \\
\hline
\end{tabular}

Banco de dados Emergency Events Revisão bibliográfica 
Database (EM-DAT- OMS) e Ministério da Saúde

Secretarias municipais de saúde

Regiões Norte (Amazônia) e Nordeste do Brasil

Secretaria Nacional de Proteção e Defesa Civil

Região Serrana do Rio de Janeiro em 2011

Defesa Civil (Ministério da Saúde do Brasil)

Análise sobre os últimos desastres ocorridos no mundo, no Brasil e em Minas Gerais

Desastres naturais registrados no município de Poços de Caldas, no Estado de Minas Gerais

Santa Catarina

Abrigo temporário implantado no município de Nova Friburgo/RJ
Elaborar uma matriz de avaliação que representasse um documento norteador para os municípios para a construção de seus planos

Estudo descritivo-exploratório de abordagem quantitativa

Estudo de caso

Estudo descritivo das informações sistematizadas e análise comparativa de abordagem quantitativa

Sistematização de dados e informações contidos no Atlas Brasileiro dos Desastres Naturais 1991-2010 e diretamente na Secretária Nacional de Defesa Civil (SNDC)

Estudo de série temporal

Pesquisa bibliográfica, inventário de dados, levantamento do material técnico, bibliográfico e cartográfico sobre a área de estudo

Estudo com desenho transversal em cinco municípios brasileiros, em duas regiões do país.

No quadro 3 foi apresentado o resumo dos estudos pesquisados, envolvendo seus cenários, método e ano.

\section{Quadro 4 - Resumo dos estudos pesquisados envolvendo objetivos e resultados.}

\begin{tabular}{|c|c|}
\hline OBJETIVOS & RESULTADOS \\
\hline $\begin{array}{l}\text { Verificar como a integração das políticas } \\
\text { Nacional de Recursos Hídricos (Lei no } \\
\text { 9.433/1997), Política Federal de Saneamento } \\
\text { (Lei nº 11.445/2007) e Política Nacional de } \\
\text { Defesa Civil (Lei no 12.608/2012) contribuem } \\
\text { para a redução das fatalidades nesses } \\
\text { desastres naturais }\end{array}$ & $\begin{array}{l}\text { Observou-se que a legislação existente é bastante } \\
\text { avançada, mas que o País carece da fiscalização } \\
\text { no cumprimento destas para efetiva redução de } \\
\text { fatalidades. Complementarmente, é necessária a } \\
\text { criação de instâncias participativas e ações } \\
\text { educativas }\end{array}$ \\
\hline $\begin{array}{l}\text { Analisar a vulnerabilidade do idoso no contexto } \\
\text { de desastres, no âmbito da Proteção e Defesa } \\
\text { Civil }\end{array}$ & $\begin{array}{l}\text { Identificou-se que o processo de urbanização } \\
\text { acelerado e desordenado das últimas décadas } \\
\text { gerou, além de outras consequências econômicas e } \\
\text { sociais, um comprometimento da infraestrutura das } \\
\text { cidades para fazer face às necessidades básicas da } \\
\text { sociedade, aumentando a vulnerabilidade de uma }\end{array}$ \\
\hline
\end{tabular}




\section{Revista FLAMMAE \\ Revista Científica do Corpo de Bombeiros Militar de Pernambuco \\ Artigo Publicado no Vol.06 N.15 - Edição Jan a Jun2020 - ISSN 2359-4829 \\ Versão on-line disponível em: http://www.revistaflammae.com}

Discutir sobre a complexidade dos desastres, realçando situações de risco e a essencialidade do suporte da Biossegurança, uma vez que, em episódios com vítimas fatais, os procedimentos formulados por este campo devem ser adotados no manejo de cadáveres, sobretudo quando da ocorrência de soterramento com busca de corpos

Desenvolver uma metodologia para avaliação dos planos de contingência elaborados pelos municípios do ERJ

Verificar exemplos de como a variabilidade climática produz impactos na saúde humana no Brasil a partir dos aspectos epidemiológicos das regiões Norte (Amazônia) e Nordeste

Analisar o planejamento de um abrigo, a partir de princípios de assistência, utilizando-se documentos da Secretaria Nacional de Proteção e Defesa Civil

Apresentar e analisar as principais bases de dados existentes sobre mortalidade por desastres ocorridos no Brasil: EM-DAT Emergency Events Database e o Atlas Brasileiro de Desastres Naturais, além do Sistema de Informações sobre Mortalidade (SIM, Ministério da Saúde) e o Anuário de Desastres Naturais (Ministério da Integração Nacional)

Analisar a inter-relação entre os desastres naturais e seus impactos sobre a saúde.

Apresentar uma síntese sobre os atuais conceitos e classificações de desastres, risco e sua relação com a vulnerabilidade ambiental e social

Estruturar uma base de dados com os levantamentos históricos e o cadastro das ocorrências de desastres naturais e dos índices pluviométricos do município de Poços de Caldas parcela da população idosa que, por falta de opção, viu-se obrigada a residir em áreas potencialmente sujeitas a enchentes, inundações e deslizamentos

O artigo traz para a análise a contribuição da Biossegurança como orientadora dos planos de emergência voltados para os desastres, realçando o risco biológico e as medidas necessárias para o manejo de cadáveres

É consenso entre o grupo técnico avaliador que a existência da matriz favoreceu o processo de análise e a elaboração de relatório de avaliação para os municípios

Um modelo conceitual de vulnerabilidade social para o desenvolvimento de estudos e intervenções nos efeitos da variabilidade climática é proposto e aplicado aos impactos de tempestades em grandes cidades, assim como à seca no Nordeste do Brasil

A infraestrutura de apoio possuía coleta de resíduos, abastecimento de água e de alimentos satisfatórios. Estava disponível equipe de profissionais de saúde. As ações de recuperação são complexas e devem estar articuladas e direcionadas ao interesse da população atingida, adaptando-se às mudanças necessárias.

A comparação mostrou que, com as bases de dados atuais, não é possível afirmar se os desastres e a mortalidade por desastres estão aumentando no país, por conta das variações dos eventos que ocorrem a cada ano

Propõe-se uma participação mais ativa do setor saúde na agenda política global pós-2015, particularmente as relacionadas ao desenvolvimento sustentável, mudanças climáticas e redução de riscos de desastres

O gerenciamento do risco deve ser uma ação prioritária e permanente nas cidades. Conforme relatado, os centros urbanos crescem cada vez mais e, muitas vezes, a forma de ocupação ou uso é inadequada ao tipo de terreno (geologia e geomorfologia)

O levantamento dos desastres naturais e a sua correlação com os registros de chuvas, associados à previsão meteorológica, subsidiam a previsão temporal e espacial da ocorrência desses eventos, com vistas a antecipar ações de defesa civil 
Revista Científica do Corpo de Bombeiros Militar de Pernambuco

Artigo Publicado no Vol.06 N.15 - Edição Jan a Jun2020 - ISSN 2359-4829

Versão on-line disponível em: http://www.revistaflammae.com

Descrever e analisar a preparação da assistência farmacêutica para desastres em municípios recentemente acometidos por desastres

Analisar comparativamente topoi do Estado e dos afetados referentes à qualidade das práticas de reabilitação implementadas como ação de defesa civil
Foram identificadas poucas medidas de preparação da assistência farmacêutica nos municípios estudados

Conclui-se que as políticas de emergência precisam avançar no reconhecimento da legitimidade da experiência e argumentos dos afetados no refazimento de sua rotina.

Todos os artigos pesquisados no quadro 4 para este estudo foram realizados a partir de dados coletados no Brasil enfatizando os principais agravos à saúde relacionados aos desastres naturais e a vulnerabilidade de populações em âmbito nacional. Os estudos selecionados foram classificados de acordo com a sua categoria de publicação, conforme explicitado pelos periódicos, assim especificados: quatro $(33,3 \%)$ pesquisas documentais; três (25\%) estudos descritivos exploratórios; três (25\%) estudos de revisão de literatura, e dois (16,6\%), estudos de caso. Em relação aos anos de publicação, compreendidos entre 2013 a 2017. Quanto à abrangência, os artigos foram assim categorizados: quatro $(33,3 \%)$ estudos qualitativos voltados para um estado, cidade ou região específicos, fundamentando-se em populações Vulneráveis e oito $(66,6 \%)$ estudos qualitativos de abrangência nacional, especificamente sob a perspectiva documental.

\section{Tipos de desastres naturais e respectivas regiões brasileiras onde ocorrem com maior frequência}

Cardoso et al (2012) relataram que os desastres naturais mais frequentes no Brasil, apontados pelo Ministério da Saúde consistiram nas inundações bruscas ou graduais, seguidas pelos escorregamentos ou deslizamentos de terra, sendo as regiões mais afetadas, a Sudeste, Sul, Nordeste, Norte e Centro-Oeste, respectivamente.

Parizzi(2014) ressaltou que o país não está fora das estatísticas mundiais e registrou mais de 900 mortes causadas por impactos das 
inundações e deslizamentos de terras provocados pela chuva, especialmente no ano de 2011. De acordo com o autor, a maior parte desses eventos, cerca de $80 \%$, está relacionada às instabilidades atmosféricas severas, que desencadeiam inundações, vendavais, tornados, granizos e escorregamentos. Normalmente, esses fenômenos são súbitos e violentos, responsáveis por grande mortandade e destruição. A Defesa Civil(2018) aponta os tipos de desastres por região brasileira (Quadro 5).

\begin{tabular}{ll} 
Quadro 5 - Distribuição de tipos de desastres por região bras \\
\cline { 2 - 2 } \multicolumn{1}{c}{ REGIÃO } & \multicolumn{1}{c}{ DESASTRE } \\
Região norte & Incêndios florestais e inundações \\
Região nordeste & Secas e inundações \\
Região centro-oeste & Incêndios florestais \\
Região sudeste & Deslizamentos e inundações \\
Região sul & Inundações, vendavais e granizo
\end{tabular}

Fonte: Defesa Civil(2018)

De acordo com os resultados do quadro 5 , as inundações foram 0 desastre mais incidente no Brasil, estando presente em todas as regiões.

\section{Regiões do Brasil mais afetadas pelos desastres naturais}

De acordo com Cardoso et al (2012), o furacão Catarina, por exemplo, em março de 2004, atingiu 26 municípios dos estados de Rio Grande do Sul e Santa Catarina, deixando 15 mil pessoas desabrigadas e 11 mortos. Em 2011, os desastres naturais tiveram um impacto significativo na sociedade brasileira, onde foi relatada cerca de 795 ocorrências, causando 1.094 óbitos e afetando 12.535.401 pessoas; 2.370 municípios foram afetados, sendo que $65,44 \%$ foi por eventos hidrológicos(PARIZZI, 2014). Na Figura 1, as regiões mais afetadas por desastres naturais. 
Figura 1- Regiões mais afetadas por desastres naturais em 2011

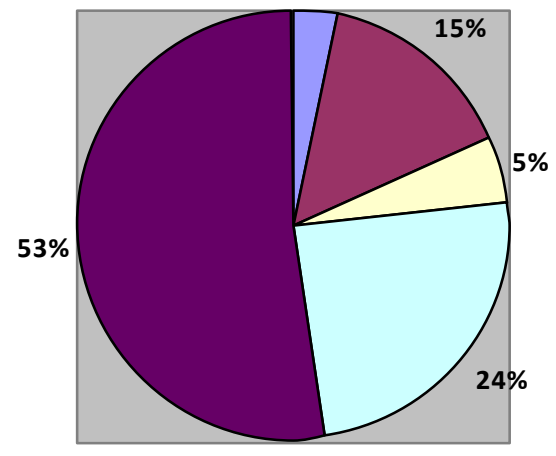

$\square$ Região Norte (77)

$\square$ Região Nordeste (355)

$\square$ Região Centro-oeste (122)

$\square$ Região Sudeste (569)

— Região Sul (1247)

Fonte: Dados do estudo

A Figura 1 mostrou que a região mais afetada foi a Região Sul com 1247 municípios atingidos. Diante desses dados, vale destacar um aspecto relevante relacionado a períodos e regiões mais críticas para cada tipo de desastre. Estes aspectos estão relacionados à distribuição dos danos humanos, com uma quantidade significativa de óbitos, observando-se na região sudeste a ocorrencia de 7,29 vezes de mais agravos quando comparados com os verificados nas outras quatro regiões, em razão do evento ocorrido na região Serrana do Rio de Janeiro no mesmo ano. Esses desastres também resultam da ocupação frequente e desordenada do solo em áreas não edificáveis no Brasil. O inchamento das cidades é igualmente responsável pelo aumento da vulnerabilidade às enchentes, enxurradas, alagamentos e outros processos que resultam em agravos significativos à saúde pública. Ressalta-se ainda que, em geral, as situações de risco mantêm correlação com a pobreza e ineficácia de políticas de desenvolvimento humano(PARIZZI, 2014).

O estudo proposto por Sardinha et al(2016)realizou um levantamento histórico e o cadastro de ocorrências de desastres naturais no município de Poços de Caldas, no estado de Minas Gerais, região conhecida por apresentar elevados índices pluviométricos e contexto geológico e geomorfológico diversificado. Entre os anos de 2000 a 2013, 25 ocorrências relacionadas a 
desastres naturais foram cadastradas, especialmente queda de barreiras, seguida por inundações, alagamentos e escorregamentos/deslizamentos.

\section{Principais agravos à saúde provocados pelos desastres naturais}

As graves consequências dos desastres de causas naturais ocorridos nos últimos anos apontam consideráveis falhas nos sistemas de segurança, principalmente em países em desenvolvimento, como o Brasil. Bodstein et al (2014) afirmam que a maioria das 3,3 milhões de mortes causadas por desastres nos últimos 40 anos, ocorreram em nações pobres, que sofrem longos períodos com as consequências desses eventos, uma vez que não possuem capacidade de recuperação rápida, o que agrava ainda mais as condições de vulnerabilidade.

De acordo com Sardinha et al(2016) os desastres naturais resultam em vítimas fatais e perdas econômicas associadas, o que torna necessárias ações de prevenção, mitigação, preparação, resposta e recuperação, voltadas à proteção dessas populações. Um plano preventivo de defesa civil (PPDC) é fundamental para a implementação de medidas que criem condições para a convivência em situações de risco, em níveis relativamente seguros para os municípios afetados. No quadro 6 , os principais agravos decorrentes de desastres naturais.

Quadro 6 - Principais fatores que contribuem para os agravos à saúde decorrentes dos desastres naturais

\section{PRINCIPAIS AGRAVOS}

Áreas inabitáveis por períodos significativos

Comprometimento dos serviços de saneamento ambiental

Comprometimento da quantidade e a qualidade da água

Comprometimento do solo e oferta de alimentos afetados

Alterações nos ciclos de vetores hospedeiros e reservatórios de doenças 
Maior incidência de doenças e óbitos

Perdas econômicas

Fonte: Defesa Civil(2018)

O estudo proposto por Valencio et al(2015)ressalta que os desastres naturais atuam como importante fator de rompimento das condições do espaço coletivo, levando o ser humano a experimentar condições conflitantes e desafiadoras. Essas conclusões resultam de um estudo de caso realizado pelos autores tendo como foco o abrigo temporário implantado no município de Nova Friburgo/RJ no ano de 2011, quando a população local foi atingida por enchentes e múltiplos deslizamentos. Um levantamento realizado por Carmo et al (2014)mostrou a relevância do megadesastreocorrido em Nova Friburgo, que atingiu de diferentesformas os diversos grupos etários em ambos os sexos, conforme o grupo 5 a 9 anos, além dos de 20 a 24 anos (paramulheres) e 30 a 34 (para homens). A partir dos dados analisados, o estado do Rio de Janeiro se destaca pelo seu histórico relacionado aos desastres, totalizando $22,98 \%$ da população total do Rio de Janeiro afetada, com 854 óbitos, no período de 19912010. Apenas em 2011, com o megadesastre, foram totalizados 858 óbitos, número acentuado quando comparado à média em 15 anos (1996-2010), de 5 óbitos no estado.

Corroborando com as ideias deValencio et al (2015) e Carvalho et al (2016), num estudo também realizado no Rio de Janeiro, ressaltam que, para se ter uma resposta efetiva nas emergências de saúde pública resultantes de um desastre, é necessário levar em conta o desenvolvimento de ações de fortalecimento das unidades técnicas, o que inclui as unidades de saúde e suas estratégias para gerenciamento de risco. Para os autores, os sistemas de vigilância epidemiológica e seus instrumentos de monitoramento e controle de doenças devem adequar-se à fácil aplicação em situações de desastres naturais. Desse modo, são necessárias instalações rápidas de postos de vigilância de doenças e o uso de formulários práticos de notificação baseados 
em sintomas e síndromes encontrados entre as vítimas.

Carvalho et al(2016), ressaltam que durante as fases apontadas para gerenciamento dos desastres naturais, o sistema de vigilância epidemiológica e controle de doenças precisa contar com o apoio de um laboratório de saúde pública para ter êxito no diagnóstico dos agravos à saúde provenientes desses eventos. Dessa maneira, é fundamental que na fase de preparo, tanto os laboratórios de referência nacional como as redes de laboratórios estaduais e municipais, sejam fortalecidos e mantenham uma rede de apoio entre si.

Dentre os principais agravos à saúde nos casos já citados de desastres naturais, o relato de experiência citado por Costa et al(2017), acerca do planejamento dos abrigos temporários em São José do Rio Preto, na catástrofe de 11 de janeiro de 2011, concorda com o descrito por Carvalho et al(2016), ao enfatizar as complexas ações de recuperação que devem estar articuladas e direcionadas ao interesse da população atingida. Nesse sentido, ambos os estudos afirmam que os profissionais de saúde devem também estar preparados para lidar com as adaptações provenientes dos desastres naturais.

Confalonieri(2015)complementa que os agravos à saúde causados pelos fenômenos climáticos no Brasil são responsáveis pela incidência de várias doenças infecciosas endêmicas que são sensíveis às variações do clima, em especial aquelas transmitidas por vetores com veiculação hídrica. Esses eventos podem causar o surgimento de novas patologias ou ainda doenças reemergentes como ocorre com a dengue. Além disso, Costa et al(2017), destacam que a interrupção e/ou contaminação dos postos e abastecimentos de água e o saneamento tendem a comprometer esses serviços por períodos prolongados, afetando a qualidade dos alimentos consumidos, seja pela população geral ou em abrigos organizados. A falta de controle nesses serviços pode resultar em surtos epidêmicos.

Os estudos de Carvalho et al(2016)enfatizam ainda que os desastres naturais podem acarretar desordem na organização dos serviços de saúde, interrompendo total ou parcialmente ações desenvolvidas por programas de 
saúde pública, seja por problemas relacionados a estrutura física das unidades, nos recursos materiais ou ainda pelo impacto nos recursos humanos alocados nos mesmos. Assim, a população já afetada pelo evento passa a estar ainda mais vulnerável.

Na mesma linha de pensamento, Cardoso et al(2012), apontam que à complexidade dos desastres naturais, das situações de risco e agravos à saúde, necessitam do suporte da biossegurança. Isso porque, em episódios com vítimas fatais, os procedimentos formulados por este campo devem estar também relacionados aos riscos biológicos em decorrência do soterramento e busca de corpos.

Ao sublinhar os desastres naturais como fenômenos imprevisíveis, súbitos e violentos, os autores enfatizam a contribuição dos elementos de biossegurança como ferramentas orientadoras dos planos de emergência, tendo em vista a necessidade de medidas necessárias para o manejo de cadáveres(CARDOSO, 2012). Uma preparação sobre assistência farmacêutica no contexto dos desastres naturais no Brasil é apontada por(SOUZA et al, 2014) como um aspecto significativo. Isso porque, o medicamento está presente em todos os níveis de cuidado em saúde e seu provimento e gestão em caso de desastre é prioritário.

Por fim, Assumpção et al(2017) ressaltam que as disponibilizações de informações sobre os agravos resultantes dos desastres naturais são importantes para a atuação dos governos e demais instituições, delineando ações de prevenção e potencializando as situações de enfrentamento do pósdesastre. Entretanto, Freitas et al (2014), enfatizam que os desastres naturais ainda são poucopesquisados e compreendidos no âmbito da saúde pública no país, com impactos do curto aos longos prazos.

\section{CONCLUSÃO}

Os desastres naturais devem ser entendidos a partir de abordagens 
complexas, contemplando um amplo diálogo com múltiplos campos do conhecimento, sobretudo, na área da saúde. Esse campo de conhecimento, tem como finalidade, a operacionalização de procedimentos voltados a mitigar riscos, contribuindo para procedimentos preventivos aos agravos à saúde. Já que, a vivência de repetidos desastres, com suas consequências ambientais, socioeconômicas e sanitárias contribuem para uma sobreposição de riscos à saúde para as populações afetadas.

Ressalta-se ainda a necessidade de sistematização de dados e informações acerca desses desastres colaborando com a implementação de políticas que possam contribuir para a redução das fatalidades ocasionadas por esses eventos. Deve-se observar também que a legislação existente é significativamente avançada, mas que o país carece da fiscalização no cumprimento destas para efetiva redução de agravos à saúde proveniente de desastres naturais.

A natureza interdisciplinar e intersetorial dos desastres necessariamente obriga a Saúde Coletiva a ter uma ampla visão sobre os mesmos, bem como a formular políticas e ações que atuem diretamente sobre seus determinantes socioambientais. Complementarmente, é necessária a criação de instâncias participativas e ações educativas.

A identificação de áreas com potenciais riscos a desastres naturais deve incentivar ações estruturais, preparação técnica de profissionais de saúde e/ou conscientização da população. Portanto, este estudo pode contribuir com informações relevantes para sociedade e profissionais de saúde, na elaboração de estratégias, ações de vigilância, prevenção e mitigação dos riscos e agravos em populações vulneráveis aos desastres.

\section{REFERÊNCIAS}

ASSUMPÇÃO, R.F. et al. Possíveis contribuições da integração das políticas públicas brasileiras à redução de desastres. Saúde em Debate, 2017; v.41, 3949, 2017. 
BODSTEIN, A. et al. A vulnerabilidade do idoso em situações de desastres: necessidade de uma política de resiliência eficaz. Ambiente \& Sociedade, v.17, n.2, 157-74, 2014.

BRASIL. Ministério da Integração Nacional, Secretaria Nacional de Defesa Civil, \& Centro Nacional de Gerenciamento de Riscos e Desastres. Anuário brasileiro de desastres naturais, 2012.

CARDOSO, T.A.O. et al. Biossegurança e desastres: conceitos, prevenção, saúde pública e manejo de cadáveres. Physis-Revista de Saúde Coletiva, v.22, n.4, 1523-42, 2012.

CARMO, R.L.; ANAZAWA, T.M. Mortalidade por desastres no Brasil: o que mostram os dados. Ciência \& Saúde Coletiva, v.19, n.9, 3669-81, 2014.

CARVALHO, S. et al. Desenvolvimento de instrumento para avaliação dos planos municipais de contingência de desastres naturais no Estado do Rio de Janeiro. Revista Rede de Cuidados em Saúde, v.10, n.3, 2016.

CONFALONIERI, E.C.U. Variabilidade climática, vulnerabilidade social e saúde no Brasil. Terra livre, v.1, n.20, 193-204, 2015.

COSTA, F.G. et al. Abrigos temporários em desastres: a experiência de São José do Rio Preto, Brasil. Saúde em Debate, v.41, 327-37, 2017.

CROSSETTI, M.G.O. Revisão integrativa de pesquisa na enfermagem: o rigor cientifico que the é exigido. Revista gaúcha de enfermagem, v.33, n.2, 8-9, 2012.

DEFESA CIVIL. Boletim Estadual de Defesa Civil. Minas Gerais: Defesa Civil; 2013.Disponível em: http://www.defesacivil.mg.gov.br/index.php/manuaisapostilas. Acesso em Mai. de 2018.

FREITAS, C.M. et al.Desastres naturais e saúde: uma análise da situação do Brasil. Ciência \& Saúde Coletiva, v.19, n.9, 3645-56, 2014.

GUIMARÃES, R.M. et al. Análise temporal da relação entre leptospirose e ocorrência de inundações por chuvas no município do Rio de Janeiro, Brasil, 2007-2012. Ciência \& Saúde Coletiva, v.19, n.9, 3683-92, 2014. 
MINERVINO, A.C.; DUARTE, E.C. Danos materiais causados à Saúde Pública e à sociedade decorrentes de inundações e enxurradas no Brasil, 2010-2014: dados originados dos sistemas de informação global e nacional. Ciência Saúde Coletiva, v.21, n.3, 685-94, 2016.

PARIZZI, M.G. Desastres naturais e induzidos e o risco urbano. Revista Geonomos, v.22, n.1, 2014.

SARDINHA, D.S. et al. Base de dados de desastres naturais no município de Poços de Caldas/MG: ferramenta para o planejamento e a gestão territorial. Urbe. Rev Bras Gest Urbana, v.8, n.3, 318-31, 2016.

SOUZA, P. et al. Preparedness of pharmaceutical assistance for disasters: a study in five Brazilian municipalities. Ciência \& Saúde Coletiva, v.19, n.9, 3731-42, 2014.

VALÊNCIO, N.F.L.S et al. O desastre como desafio para construção de uma hermenêutica diatópica entre o Estado e os afetados. Revista Cronos, v.8, n.1, 81-100, 2015. 